\title{
Association between Asymptomatic Unilateral Internal Carotid Artery Stenosis and Electrophysiological Function of the Retina and Optic Nerve
}

\author{
Anna Machalińska, ${ }^{1}$ Aleksandra Kowalska-Budek, ${ }^{1}$ Miłosz Piotr Kawa, \\ Arkadiusz Kazimierczak, ${ }^{3}$ Krzysztof Safranow, ${ }^{4}$ Marta Kirkiewicz, ${ }^{1}$ Grażyna Wilk, ${ }^{5}$ \\ Wojciech Lubiński, ${ }^{1}$ Piotr Gutowski, ${ }^{3}$ and Bogusław Machaliński ${ }^{2}$ \\ ${ }^{1}$ Department of Ophthalmology, Pomeranian Medical University, Szczecin, Poland \\ ${ }^{2}$ Department of General Pathology, Pomeranian Medical University, Szczecin, Poland \\ ${ }^{3}$ Department of Vascular Surgery and Angiology, Pomeranian Medical University, Szczecin, Poland \\ ${ }^{4}$ Department of Biochemistry and Medical Chemistry, Pomeranian Medical University, Szczecin, Poland \\ ${ }^{5}$ Department of General and Dental Radiology, Pomeranian Medical University, Szczecin, Poland
}

Correspondence should be addressed to Anna Machalińska; annam@pum.edu.pl and Bogusław Machaliński; machalin@pum.edu.pl

Received 1 December 2016; Revised 9 February 2017; Accepted 12 March 2017; Published 12 April 2017

Academic Editor: Takaaki Hayashi

Copyright (C) 2017 Anna Machalińska et al. This is an open access article distributed under the Creative Commons Attribution License, which permits unrestricted use, distribution, and reproduction in any medium, provided the original work is properly cited.

Purpose. This study was designed to assess retinal and optic nerve bioelectrical function in patients with unilateral asymptomatic but hemodynamically significant internal carotid artery stenosis (ICAS). Methods. Forty-two subjects with a diagnosis of unilateral ICAS and 34 controls were analyzed. Full-field electroretinogram (ERG), pattern electroretinogram (PERG), and pattern visual-evoked potentials, as well as optical coherence tomography and ophthalmological examination, were performed. Data analysis included eyes ipsilateral to ICAS (EIS) and eyes contralateral to ICAS (ECS). Results. Intraocular pressure was significantly decreased in EIS and ECS compared to that in the controls. In the macula, both the cube average thickness and cube volume values were significantly reduced both in EIS and ECS compared to those in the controls. Similarly, PERG P50 and N95 wave amplitudes were significantly smaller in EIS and ECS compared to those in the controls. The ERG rod b-wave and rod-cone a-wave amplitudes were decreased, and implicit times were significantly prolonged, whereas the OP wave index was reduced in EIS compared to that in the controls. No differences in IOP, OCT, or ERG and PERG parameters were identified between EIS and ECS. Conclusions. Our study demonstrated that retinal bioelectrical function is negatively affected by ICAS despite the absence of objective clinical signs and symptoms of ocular ischemia.

\section{Introduction}

The management of patients with asymptomatic carotid artery stenosis is one of the most controversial problems in clinical practice. The outcomes of the Asymptomatic Carotid Atherosclerosis Study (ACAS) have indicated that carotid endarterectomy (CEA) reduces the risk of stroke compared with noninvasive management [1]; however, the potential risks of angiography and vascular surgical intervention may not justify their introduction into routine clinical strategies. The benefit of the CEA procedure may be meaningfully increased in patients with a high predisposition to develop cerebrovascular events [2]. To date, defining a high-risk patient with asymptomatic severe ICAS remains 
problematic, and it is necessary to establish reliable tests that may be used to identify hemodynamically impaired patients, particularly in the subclinical phase of ischemia.

The retina is a light-sensitive tissue of the central nervous system and is composed of several types of cells. The functional signals and activities of each cell type may be recorded via electroretinography (ERG). ERG is a sensitive tool used to detect ischemic injury, and it is well established how retinal ischemia may affect different retinal cells and their functions. The b-wave of flash ERG is a particularly sensitive index of retinal ischemia and represents a functional measure for retinal ischemic damage as a result of the reduction of blood flow or blood pressure [3]. Accordingly, the oscillatory response has been suggested to represent a valid measure of the functional integrity of the microcirculation of the inner retina [4]. Moreover, pattern ERG (PERG) has the ability to quantify retinal ganglion cell function in the course of ischemia [5].

The clinical associations between retinal ischemia and internal carotid artery stenosis (ICAS) are well established. The ophthalmic manifestations of carotid insufficiency encompass a heterogeneous spectrum of clinical manifestations that varies according to the ICAS extent and its duration, including transient episodes of monocular blindness (referred to as amaurosis fugax), venous stasis retinopathy, ocular ischemic syndrome with rubeosis iridis, and the development of neovascular glaucoma [6]. The most common ocular symptom, which occurs in $30-40 \%$ of patients with atherosclerotic carotid occlusive disease, is ipsilateral transient visual loss [7]. More than $90 \%$ of patients with transient monocular visual loss have abnormal carotid angiograms [8]. Furthermore, affected patients are also at risk for developing ocular/orbital pain, aqueous flare, and cataracts. Fluorescein angiography has demonstrated delayed and patchy choroidal filling and diffuse leakage from the retinal vessels and the optic nerve head [9]. Interestingly, ocular signs and symptoms may represent the first manifestation of carotid stenosis [7].

It is worth noting that in general, the retinal examination of patients with ICAS is performed in direct time relation to the occurrence of clinical ocular symptoms. However, we hypothesize that prior to the development of the clinical ocular symptoms associated with ICAS, the choroid and retina may be affected by ischemia that results in subclinical electrophysiological retinal dysfunction. It may even represent its unique detectable manifestation. The incidence of abnormal retinal functioning in asymptomatic ICAS in the course of atherosclerotic carotid artery disease has previously been investigated using a full-field ERG and multifocal ERG (mfERG) to a limited extent [10, 11]. As ERG and mfERG originate from preganglionic elements [12], these electrophysiological tests do not enable the functional evaluation of the innermost retinal layers, particularly the macular region. In contrast, the function of ganglion cells and their fibers may be specifically assessed by pattern electroretinogram (PERG) and pattern visual evoked potentials (PVEP) [13]. Nevertheless, PERG and PVEP results in the eyes of patients with significant ICAS are currently unknown.
Therefore, the present study is designed to assess retinal and optic nerve bioelectrical function using ERG, combined with PERG and PVEP in both eyes of patients with unilateral asymptomatic but hemodynamically significant ICAS without objective signs and symptoms of ocular ischemia.

\section{Material and Methods}

2.1. Characteristics of the Study Group. Eighty-four eyes of 42 subjects with a diagnosis of unilateral hemodynamically significant ICAS (i.e., $\geq 70 \%$ of ICA diameter reduction in neurologically asymptomatic patients, defined as subjects, who lack chronic or transient ischemic symptoms typical for the carotid territory or transient ipsilateral blindness in the previous six months prior to study recruitment) were analyzed. The typical carotid territory ischemic symptoms included contralateral weakness of the face, arm, leg, or both and contralateral sensory deficit or paresthesia of the face, arm, leg, or both as a result of a developed stroke or transient ischemic attack (TIA) [14]. The subjects were randomly selected from a group of patients in the Vascular Surgery Department of Pomeranian Medical University in Szczecin, Poland who qualified for carotid endarterectomy (CEA). Thirty-four age- and sex-matched volunteers without signs of carotid stenosis or exceptionally with ICAS at a grade lower than $40 \%$ were enrolled as the control group. The degree of each individual's ICAS was diagnosed via carotid color Doppler ultrasonography (CDU) using a Voluson 730 PRO, GE Medical Systems device (Milwaukee, WI, USA), with a multifrequency $7.5-\mathrm{MHz}$ linear probe, in accordance with the guidelines recommended in the literature $[15,16]$. Patients with ocular ischemic syndrome or evidence of concomitant eye and chronic systemic disease that potentially affects the retina and optic nerve, that is, glaucoma, intraocular inflammatory diseases, retinopathy, recent (within 3 months) ocular surgery, advanced cataract, collagen-related or neoplastic disease, or a history of previous contralateral/ unilateral CEA or ICAS stenting were excluded from the study. All enrolled subjects underwent a complete ophthalmic examination of both eyes, that is, distant best-corrected visual acuity (DBCVA), intraocular pressure (IOP) measurements, and anterior segment and dilated ophthalmoscopy fundus examinations using slit lamp biomicroscopy. The ophthalmological inclusion criteria included a bestcorrected distance visual acuity (VA) $>0.8$ (Snellen chart) and normal results with respect to a routine ophthalmological examination.

Data regarding medical history, current drug use, and smoking status were collected based on laboratory data, pathology tests, and other information, with particular focus on heart and vascular conditions and preexisting arterial hypertension. Furthermore, the actual arterial blood pressure (BP) was directly measured prior to ophthalmic examination in all subjects using a noninvasive blood pressure system with a manual aneroid manometer. The mean result from three measurements obtained with 5-minute-resting intervals was calculated. From the obtained BP data, the systemic mean arterial pressure (MAP) was calculated as follows: $\mathrm{MAP}=$ diastolic $\mathrm{BP}+1 / 3$ (systolic $\mathrm{BP}-$ diastolic $\mathrm{BP}) \mathrm{mmHg}$. 
Furthermore, the following medical parameters were assessed in all patients: waist circumference $[\mathrm{cm}]$, waist/hip ratio (WHR), and body mass index (BMI) [weight $(\mathrm{kg}) / \mathrm{height}$ (m)2]. Cumulative pack-years were calculated using the reported average number of cigarettes smoked per day and the number of years of smoking.

The study adhered to the tenets of the Declaration of Helsinki, and approval was obtained from the Local Research Ethics Committee. Moreover, each patient provided written informed consent for his or her involvement.

2.2. Electrophysiology. Full-field electroretinograms (UTAS-E 2000 system, LKC Technologies, Inc.), pattern electroretinograms, and pattern visual evoked potentials (RetiPort System, Roland Consult Instr.) were recorded according to the International Society for Clinical Electrophysiology of Vision (ISCEV) protocols [17-19]. For full-field ERG, recordings were performed binocularly with the use of bipolar BurianAllen electrodes (Hansen Ophthalmic Development Lab, Iowa, USA) and a ground clip gold electrode (Natus Europe $\mathrm{GmbH}$, Planegg, Germany) attached to the earlobe. Prior to recording, the cornea was anesthetized (Alcaine; Alcon) and the pupils were dilated with $1 \%$ tropicamide eye drops. The recording system parameters were as follows: amplifier sensitivity, $10-20-50 \mu \mathrm{V} / \mathrm{div}$; filters, $0.3-500 \mathrm{~Hz}$ (for OP extraction: 75-500 Hz); notch filters, off; time base, $5 \mathrm{~ms} / \mathrm{div}$; and artifact reject threshold, $0 \mu \mathrm{V}$. A $30 \mathrm{~min}$ dark adaptation preceded the rod and mixed cone-rod recordings; a $10 \mathrm{~min}$ period of light adaptation preceded the photopic cone and flicker recordings. The rod ERG recordings were obtained with single dim white flashes of $1.6 \mathrm{~cd} \mathrm{~s} \mathrm{~m}^{-2}$ attenuated by a $24 \mathrm{~dB}$ neutral filter. The mixed rod-cone ERGs were elicited with flashes of white light of $1.6 \mathrm{~cd} \mathrm{~s} \mathrm{~m}^{-2}$. The cone ERGs were elicited with white flashes of the same intensity. In the flicker recordings, the frequency of stimulation was $30 \mathrm{~Hz}$ (the same conditions of adaptation) and ten sweeps were averaged. The oscillatory potentials in the scotopic state were obtained with $1.6 \mathrm{~cd} \mathrm{~s} \mathrm{~m}^{-2}$ white flashes. The overall wave index of the $\mathrm{OP}$ amplitudes $(\mathrm{O} 1+\mathrm{O} 2+\mathrm{O} 3)$ was measured.

For PERG, monocular stimulation was used, in combination with an appropriate refractive error correction in relation to the eye-screen distance. The examination was interrupted when frequent blinking or fixation losses were identified (the patient was monitored with a TV camera). The pupils were not dilated, and central fixation was used. The PERG stimulation parameters were as follows: a $21^{\prime \prime}$ CRT monitor with a frame rate equal to $70 \mathrm{fps}$ was used; dimension of the stimulus field was $15024^{\prime}$ (the mean of the width and the height of the screen), with the aspect ratio between the width and the height (screen proportion $\mathrm{H} / \mathrm{V}$ ) equal to 4:3; black and white reversing checkerboard was presented to the patient, with a check size equal to 0048'; luminance for white elements was equal to $120 \mathrm{~cd} / \mathrm{m}^{2}$, mean luminance of the stimulus screen: $62 \mathrm{~cd} / \mathrm{m}^{2}$, with Michelson contrast set to $97 \%$; temporal frequency was equal to $4.0 \mathrm{rps}$ $(2.0 \mathrm{~Hz})$. The electrodes were as follows: a ground (gold disk) electrode (Roland Consult, Brandenburg, Germany) was placed on the forehead (Fpz), a thread Dawson-Trick-
Litzkow (DTL) electrode (Diagnosys LLC, MA, USA) was used as active, and a gold disk placed at the ipsilateral outer canthus was used as a reference. The recording system parameters were as follows: amplifier sensitivity $-20 \mu \mathrm{V} / \mathrm{div}$, filters: $1-100 \mathrm{~Hz}$, artifact reject threshold $-95 \%$ (for the amplifier range $\pm 100 \mu \mathrm{V}$ ), notch filters were off, averaging: 200 sweeps, and sweep time: $250 \mathrm{~ms}$ (time base: $25 \mathrm{~ms} /$ div). Two consecutive waveforms were recorded; they were subsequently averaged and analyzed off-line.

For pattern visual evoked potentials, monocular stimulation was used without pupil dilation and refraction correction was applied with respect to the eye-screen distance. Central fixation was used. Patient was monitored with a TV camera, and interruptions of the test were introduced when fixation loose or frequent blinking was observed. $21^{\prime \prime}$ CRT monitor with a frame rate equal to $70 \mathrm{fps}$ was used for pattern stimulation; dimension of the stimulus field was 170 (the height of the screen), with aspect ratio between the width and the height (screen proportion $\mathrm{H} / \mathrm{V}$ ) equal to 4 :3; black and white reversing checkerboard was presented to the patient, with two check sizes equal to $0016^{\prime}$ and $104^{\prime}$; mean luminance of the stimulus screen was equal to $60 \mathrm{~cd} / \mathrm{m}^{2}$, (luminance for white elements: ca. $120 \mathrm{~cd} / \mathrm{m}^{2}$ ), with Michelson contrast set to $97 \%$; temporal frequency was equal to $1.875 \mathrm{rps}(0.938 \mathrm{~Hz})$. The electrodes were as follows: an active-gold disk electrode (Roland Consult, Brandenburg, Germany) was placed on the scalp over the visual cortex at $\mathrm{Oz}$ with a reference-gold disk electrode placed at $\mathrm{Fz}$; a ground (gold disk) electrode was placed on the forehead at fpz. Before recordings, interelectrode impedance was measured; values $<5 \mathrm{k} \Omega$ were accepted. Parameters of the recording channel were as follows: amplifiers range $\pm 100 \mu \mathrm{V} / \mathrm{div}$, filter frequency bandwidth 1-100 Hz. Analysis period (sweep time) was equal to $300 \mathrm{~ms}$. Artifact reject threshold was set to $95 \%$, and 100 sweeps were averaged. For each eye and each check size, two consecutive PVEP waveforms were recorded and off-line averaged for further analysis.

2.3. Optical Coherence Tomography. The macular and peripapillary retinal nerve fiber layer (RNFL) thicknesses were measured before and 3 months after endarterectomy for each patient, using optical coherence tomography (Cirrus OCT, Zeiss Humphrey System, Dublin, California, USA). A macular profile of the central $6 \mathrm{~mm}$ zone was obtained using a fast macular scan protocol. The central subfield thickness (CST), cube average thickness (CAT), and cube volume (CV) automatically measured by a retinal border detection algorithm in the software were used for statistical analysis.

2.4. Statistical Analysis. Parameters were compared between independent groups with the Mann-Whitney test for quantitative variables and Fisher's exact test for qualitative variables. The Wilcoxon signed-rank test was used to compare parameters between the eyes ipsilateral and contralateral to ICAS. The strength of the association between the quantitative variables was measured with Spearman rank correlation coefficient (Rs). A $p<0.05$ was considered statistically significant. 
TABLE 1: Clinical characteristics of the study groups.

\begin{tabular}{|c|c|c|c|}
\hline Parameter & ICAS group & Control group & $p$ value $^{\mathrm{a}}$ \\
\hline Number of subjects & 42 & 34 & - \\
\hline Sex (male/female) & $28 / 14$ & $21 / 13$ & 0.81 \\
\hline Patient's age [years] $($ mean $\pm \mathrm{SD})$ & $62.5 \pm 6.5$ & $62.5 \pm 4.0$ & 0.80 \\
\hline $\mathrm{BMI}\left[\mathrm{kg} / \mathrm{m}^{2}\right]($ mean $\pm \mathrm{SD})$ & $27.91 \pm 3.67$ & $27.34 \pm 3.63$ & 0.30 \\
\hline WHR [arbitrary units] (mean \pm SD) & $0.94 \pm 0.11$ & $0.93 \pm 0.11$ & 0.52 \\
\hline Waist circumference $[\mathrm{cm}]($ mean $\pm \mathrm{SD})$ & $97.24 \pm 13.31$ & $97.47 \pm 13.24$ & 0.98 \\
\hline MAP $[\mathrm{mmHg}]($ mean \pm SD) & $104.30 \pm 9.87$ & $104.05 \pm 11.07$ & 0.96 \\
\hline Current smokers (\%) & 33.33 & 23.53 & 0.45 \\
\hline Former smokers (\%) & 85.71 & 76.47 & 0.38 \\
\hline Smoking pack-years (mean \pm SD) & $31.05 \pm 30.94$ & $27.18 \pm 24.48$ & 0.67 \\
\hline Period without smoking [years] $($ mean $\pm S D)$ & $5.36 \pm 6.86$ & $7.40 \pm 10.59$ & 0.99 \\
\hline Hypertension (\%) & 80.95 & 64.71 & 0.12 \\
\hline Duration of hypertension [years] $($ mean $\pm \mathrm{SD})$ & $5.66 \pm 6.06$ & $7.68 \pm 8.51$ & 0.75 \\
\hline History of ischemic heart disease (\%) & 35.71 & 20.59 & 0.20 \\
\hline History of cardiac infarction (\%) & 16.67 & 17.65 & 1.00 \\
\hline History of cerebral stroke (\%) & 33.33 & 8.82 & 0.01 \\
\hline History of peripheral artery disease (\%) & 23.81 & 23.53 & 1.00 \\
\hline History of aortic aneurysm (\%) & 9.52 & 11.76 & 1.00 \\
\hline
\end{tabular}

${ }^{\mathrm{a}}$ Mann-Whitney test for quantitative variables and Fisher's exact test for qualitative ones.

\section{Results}

Eighty-four eyes of 42 patients with significant unilateral ICAS were included. Thirty-four controls had both eyes analyzed. The eyes were organized into 3 groups: EIS-eyes ipsilateral to ICAS $(n=42)$, ECS - eyes contralateral to ICAS $(n=42)$, and controls without ICAS $(n=68)$. The mean extent of internal carotid stenosis was $79 \pm 12 \%$.

The clinical characteristics of the patients and controls are summarized in Table 1 . The ICAS and control groups were matched for age, gender, and selected, well-known atherosclerotic risk factors, including hypertension, history of ischemic heart disease, cardiac infarction, peripheral artery disease, and aortic aneurysm. The rate of previous stroke was significantly increased in the ICAS group compared with the control group (33.33\% versus $8.82 \%$, resp.). Moreover, there was no significant difference in the values of the BMI, WHR, MAP, and proportion of active and former smokers between the groups.

Table 2 provides the values of the IOP and OCT parameters obtained for all investigated groups of eyes. The IOP values were significantly smaller in the EIS and ECS compared with the controls. In the macula, both the cube average thickness and cube volume values were significantly reduced both in the EIS and ECS compared with the controls. Importantly, no significant difference was identified between the eyes from the EIS and ECS groups in the patients with ICAS. Moreover, there was no difference in the value of peripapillary RNFL in the EIS and ECS groups compared with the controls.

Table 3 indicates the values of the PERG and PVEP measurements obtained in the eyes of all investigated groups. The amplitudes of the PERG P50 and N95 waves were significantly smaller in the EIS and ECS compared with the controls. No significant difference in both amplitudes was identified between the eyes from the EIS and ECS groups in the patients with ICAS. When analyzing the VER parameters, the amplitudes and peak times of the P100 waves did not significantly differ compared with the controls.

The values of the full-field ERG amplitudes and peak times for all groups are summarized in Table 4 . The rod bwave amplitudes were significantly decreased $(p=0.01)$, and the implicit times were significantly prolonged $(p=0.0004)$ in the EIS group compared with the controls. Similarly, in the ECS group, the values of the rod b-wave implicit times were significantly prolonged $(p<0.0001)$ compared with the controls. Furthermore, in the EIS and fellow eyes, we identified a significant decrease in the rod-cone a-wave amplitudes $(p=0.05$ and $p=0.008$, resp.) and a delay in the a-wave peak times $(p=0.02$ and $p=0.007$, resp.). When the OP wave index was analyzed, a significant decrease in the amplitudes was identified exclusively in the EIS $(p=0.02)$. Remarkably, there were no differences in the full-field ERG wave amplitudes and implicit times between the EIS and ECS.

Furthermore, to characterize the other risk factors that may influence the electrophysiological function of the retina and optic nerve, we investigated the association between fullfield ERG amplitudes and peak times and several systemic risk factors and conditions. We determined that increasing the WHR was associated with longer rod b-wave implicit times ( $\mathrm{Rs}=+0.33, p=0.03$ in EIS; Rs $=+0.36, p=0.02$ in ECS; and Rs $=+0.25, p=0.04$ in controls). Thus, the WHR positively correlated with the rod-cone a-wave peak times (Rs $=+0.15, p=0.33$ in EIS; Rs $=+0.22, p=0.15$ in ECS; and $\mathrm{Rs}=+0.36, p=0.002$ in controls) and the rod-cone $\mathrm{b}$-wave 
TABLE 2: Mean values ( \pm standard deviation) of IOP and OCT parameters in EIS, ECS, and control eyes.

\begin{tabular}{lcccccc}
\hline Parameter & EIS & ECS & Control & & \multicolumn{2}{c}{$\begin{array}{c}\text { value } \\
\end{array}$} \\
& $n=42$ & $n=42$ & $n=68$ & EIS versus control $^{\mathrm{a}}$ & ECS versus control $^{\mathrm{a}}$ & EIS versus ECS $^{\mathrm{b}}$ \\
\hline IOP $(\mathrm{mmHg})$ & $15.27 \pm 2.65$ & $15.48 \pm 2.36$ & $16.57 \pm 2.49$ & $\mathbf{0 . 0 0 6}$ & $\mathbf{0 . 0 3}$ & 0.70 \\
CST $(\mu \mathrm{m})$ & $268.69 \pm 23.0$ & $265.293 \pm 21.7$ & $269.9 \pm 20.1$ & 0.87 & 0.39 & 0.62 \\
CV $\left(\mathrm{mm}^{3}\right)$ & $9.90 \pm 0.45$ & $9.89 \pm 0.52$ & $10.06 \pm 0.43$ & $\mathbf{0 . 0 4}$ & $\mathbf{0 . 0 3}$ & 0.64 \\
CAT $(\mu \mathrm{m})$ & $276.95 \pm 12.72$ & $276.78 \pm 14.42$ & $281.81 \pm 11.99$ & $\mathbf{0 . 0 3}$ & 0.03 & 0.27 \\
RNFL thickness $(\mu \mathrm{m})$ & $91.50 \pm 8.30$ & $92.10 \pm 10.46$ & $93.72 \pm 8.78$ & 0.21 & 0.50 \\
\hline
\end{tabular}

${ }^{\mathrm{a}}$ Mann-Whitney test; ${ }^{\mathrm{b}} \mathrm{W}$ ilcoxon signed-rank test.

TAble 3: Mean values ( \pm standard deviation) of PERG and PVEP amplitudes and peak times in EIS, ECS, and control eyes.

\begin{tabular}{|c|c|c|c|c|c|c|}
\hline \multirow{2}{*}{ Parameter } & \multirow{2}{*}{$\begin{array}{c}\text { EIS } \\
n=42\end{array}$} & \multirow{2}{*}{$\begin{array}{c}\text { ECS } \\
n=42\end{array}$} & \multirow{2}{*}{$\begin{array}{c}\text { Control } \\
n=68\end{array}$} & \multicolumn{3}{|c|}{$p$ value } \\
\hline & & & & EIS versus control $^{\mathrm{a}}$ & $\mathrm{ECS}$ versus control ${ }^{\mathrm{a}}$ & EIS versus $\mathrm{ECS}^{\mathrm{b}}$ \\
\hline \multicolumn{7}{|l|}{ PERG } \\
\hline N35-P50 amplitude $(\mu \mathrm{V})$ & $2.78 \pm 1.67$ & $2.63 \pm 2.02$ & $3.65 \pm 1.51$ & 0.01 & 0.0002 & 0.19 \\
\hline P50 peak time (ms) & $53.87 \pm 4.95$ & $52.64 \pm 4.13$ & $54.19 \pm 3.16$ & 0.47 & 0.03 & 0.57 \\
\hline P50-N95 amplitude $(\mu \mathrm{V})$ & $4.22 \pm 2.97$ & $3.75 \pm 2.91$ & $5.62 \pm 2.24$ & 0.002 & $<0.0001$ & 0.06 \\
\hline \multicolumn{7}{|l|}{ PVEP } \\
\hline P100 amplitude $(\mu \mathrm{V})\left(1^{\circ} 4^{\prime}\right)$ & $7.1 \pm 3.93$ & $7.21 \pm 3.97$ & $7.96 \pm 3.47$ & 0.13 & 0.20 & 0.58 \\
\hline P100 amplitude $(\mu \mathrm{V})\left(0^{\circ} 8^{\prime}\right)$ & $8.63 \pm 5.75$ & $8.4 \pm 4.8$ & $9.15 \pm 5.13$ & 0.43 & 0.43 & 0.34 \\
\hline P100 peak time $(\mathrm{ms})\left(1^{\circ} 4^{\prime}\right)$ & $112.91 \pm 10.84$ & $114.5 \pm 8.63$ & $111.23 \pm 8.48$ & 0.52 & 0.07 & 0.13 \\
\hline P100 peak time $(\mathrm{ms})\left(0^{\circ} 8^{\prime}\right)$ & $118.24 \pm 11.95$ & $119.57 \pm 11.42$ & $118.83 \pm 8.2$ & 0.63 & 0.44 & 0.65 \\
\hline
\end{tabular}

${ }^{\mathrm{a}}$ Mann-Whitney test; ${ }^{\mathrm{b}}$ Wilcoxon signed-rank test.

TABLE 4: Mean values ( \pm standard deviation) of full-field ERG amplitudes and peak times in EIS, ECS, and control eyes.

\begin{tabular}{|c|c|c|c|c|c|c|}
\hline \multirow{2}{*}{ Parameter } & \multirow{2}{*}{$\begin{array}{c}\text { EIS } \\
n=42\end{array}$} & \multirow{2}{*}{$\begin{array}{c}\text { ECS } \\
n=42\end{array}$} & \multirow{2}{*}{$\begin{array}{c}\text { Control } \\
n=68\end{array}$} & \multicolumn{3}{|c|}{$p$ value } \\
\hline & & & & EIS versus control $^{\mathrm{a}}$ & ECS versus control ${ }^{\mathrm{a}}$ & EIS versus $\mathrm{ECS}^{\mathrm{b}}$ \\
\hline \multicolumn{7}{|l|}{ Amplitude $(\mu \mathrm{V})$} \\
\hline Rod b-wave & $82.08 \pm 38.01$ & $92.02 \pm 38.99$ & $98.6 \pm 27.84$ & 0.01 & 0.096 & 0.09 \\
\hline Rod-cone a-wave & $159.46 \pm 57.3$ & $148.82 \pm 49.18$ & $177.15 \pm 48.38$ & 0.05 & 0.008 & 0.17 \\
\hline Rod-cone b-wave & $381.18 \pm 84.5$ & $380.04 \pm 88.6$ & $397.09 \pm 67.48$ & 0.26 & 0.14 & 0.84 \\
\hline OPs wave index & $98.05 \pm 28.95$ & $100.24 \pm 28.25$ & $114.46 \pm 34.86$ & 0.02 & 0.08 & 0.40 \\
\hline Cone a-wave single flash & $26.56 \pm 11.55$ & $24.51 \pm 11.81$ & $29.44 \pm 8.03$ & 0.12 & 0.01 & 0.49 \\
\hline Cone b-wave single flash & $83.87 \pm 29.17$ & $82.06 \pm 25.93$ & $89.18 \pm 22.88$ & 0.23 & 0.09 & 0.85 \\
\hline Cone b-wave $30 \mathrm{~Hz}$ flicker & $70.07 \pm 26.85$ & $66.39 \pm 26.31$ & $76.64 \pm 18.63$ & 0.07 & 0.008 & 0.13 \\
\hline \multicolumn{7}{|l|}{ Peak time $(\mathrm{ms})$} \\
\hline Rod b-wave & $112.12 \pm 10.73$ & $113.99 \pm 9.65$ & $105.71 \pm 8.03$ & 0.0004 & $<0.0001$ & 0.12 \\
\hline Rod-cone a-wave & $24.31 \pm 1.58$ & $24.32 \pm 1.6$ & $23.9 \pm 1.07$ & 0.02 & 0.007 & 0.91 \\
\hline Rod-cone b-wave & $48.1 \pm 3.45$ & $48.11 \pm 3.84$ & $47.68 \pm 2.83$ & 0.11 & 0.09 & 0.85 \\
\hline Cone a-wave single flash & $16.13 \pm 1.51$ & $16.11 \pm 1.59$ & $15.95 \pm 1.2$ & 0.74 & 0.87 & 0.96 \\
\hline Cone b-wave single flash & $32.2 \pm 1.92$ & $32.23 \pm 1.99$ & $32.03 \pm 1.64$ & 0.68 & 0.43 & 0.77 \\
\hline Cone b-wave $30 \mathrm{~Hz}$ flicker & $33 \pm 0.86$ & $33.05 \pm 0.79$ & $32.89 \pm 1.06$ & 0.82 & 0.89 & 0.99 \\
\hline
\end{tabular}

${ }^{\mathrm{a}}$ Mann-Whitney test; ${ }^{\mathrm{b}}$ Wilcoxon signed-rank test.

peak times $(\mathrm{R} s=+0.13, p=0.40$ in EIS; Rs $=+0.19, p=0.22$ in ECS; and Rs $=+0.24, p=0.04$ in controls). Interestingly, the rod b-wave and rod-cone b-wave amplitudes, as well as the magnitudes of the OP wave index, were dependent on the amount of cigarettes smoked. In the control group, an increase in the smoking pack-years was associated with a lower OP wave index (Rs $=-0.33, p=0.006)$ and lower rod b-wave (Rs $=-0.36, p=0.002)$ and rod-cone b-wave (Rs $=-0.5, p<0.0001)$ amplitudes. Similarly, the magnitudes of both the PERG P50 and N95 wave amplitudes were 
correlated with the amount of cigarettes smoked. An increase in the smoking pack-years was associated with lower PERG P50 wave amplitudes (Rs $=0.49, p<0.0001)$ and N95 wave amplitudes (Rs $=-0.33, p=0.006)$.

\section{Discussion}

Ocular circulation is predominately controlled by autoregulative mechanisms, which maintain a stable retina and, to a large extent, the choroidal and optic nerve blood supply. In the case of carotid atherosclerosis and hemodynamically significant internal carotid artery stenosis, chronic cerebral hypotension may induce a downward shift in the cerebral and ocular vascular autoregulation. A major part of the oxygen and glucose consumed by the retina is delivered by the choroid [20]. The choroid has one of the highest blood flows in the body; however, it has no effective autoregulation. Consequently, the outer retina is more susceptible to hypoxia as a result of systemic ischemia [21]. It has been demonstrated that patients with ICAS have thinner choroids compared with age-matched healthy individuals [22]. Similarly, choroidal hypoperfusion as a result of ICAS resulted in multiple occlusions of the choriocapillaris and attenuated choroidal vessels [23]. In contrast, retinal circulation through the central retinal artery is highly dependent on the blood concentration of $\mathrm{O}_{2}$ and $\mathrm{CO}_{2}$ and the $\mathrm{pH}$ levels in the inner retina $[24,25]$. Retinal flux may be increased in hypoxia by up to $336 \%$ [24], which is not possible in the choroid.

It has been demonstrated that full-field a- and b-wave ERG amplitudes are reduced in ocular ischemic syndrome compared with healthy eyes [9]. More recently, Kofoed et al. identified significant delays in all first-order summed implicit times and a clear trend towards reduced amplitudes in mfERG compared with healthy controls [26]. In the present study, we demonstrated that even in the absence of the clinical signs of ocular ischemic syndrome, significant ICAS may be associated with retinal function abnormalities. We identified significantly decreased amplitudes and prolonged peak times of scotopic b-waves and scotopic/photopic a-waves, as well as a reduced OP wave index in eyes ipsilateral to ICAS compared with controls. It is widely accepted that the a-wave is primarily generated from photoreceptors, and the b-wave is generated from bipolar cells [27]. Oscillatory potentials (OPs) are small rhythmic waves superimposed on the ascending b-wave of the ERG, and amacrine and bipolar cells are directly or indirectly involved in their generation [4]. Thus, our findings suggest that the rodmediated response is mainly affected as a result of significant carotid stenosis. It is worth noting that the outer retina is at an increased risk of hypoxic injury in the dark compared with the light because oxygen consumption in photopic conditions represents only $60 \%$ of consumption in a scotopic environment [28, 29]. Increased oxygen consumption is related to an increase in retinal blood flow from $40 \%$ to $70 \%$ following the transition from light to dark [30]. Our full-field ERG results are in agreement with the findings of other groups [10].
Our findings indicated that ICAS may cause impairment in macular function, which was demonstrated by the significant reduction in the PERG P50 and N95 wave amplitudes. PERG has clinically been used to evaluate macular function because it is a focal response that reflects the activity of directly stimulated retinal areas and is thought to be correlated mainly with ganglion cell activity [31]. The objective assessment of macular function using PERG permits the practical differentiation between maculopathy and optic neuropathy in different clinical settings [32]. To the best of our knowledge, PERG testing has not been previously used to evaluate preganglionic macular function in patients with significant carotid stenosis. The previously published study performed with the use of mfERG in patients with unilateral carotid artery stenosis and without clinical eye disease demonstrated significantly reduced and delayed response in electroretinogram, which was correlated with an increasing grade of carotid artery stenosis [11]. Interestingly, cone function deviations have been demonstrated to correlate with actual arterial blood pressure [11]. These findings may suggest that the macula is affected well before the onset of clinical eye disease. Our results indicate the importance of PERG testing in the evaluation and management of patients with asymptomatic severe ICAS to identify macular dysfunction as a result of ICASrelated ischemic conditions.

Interestingly, impaired macular function was accompanied by decreased macular thickness and volume compared with the control group as detected in SD-OCT images in our study. Thus, we speculate that a reduction in PERG wave amplitudes is associated with a permanent loss of retinal cells in the macular region. Sepah et al. demonstrated a strong relationship between retinal function and the thicknesses of individual retinal layers based on the correlation between retinal parameters on OCT images and functional loss detected in ERG [33]. Interestingly, we identified reduced intraocular pressure in eyes ipsilateral and contralateral to ICAS compared with controls. This finding is in concordance with a previous report, in which carotid artery stenosis reduced the blood supply to the eye, which significantly affected several blood flow parameters, such as the intraocular pressure, ocular systolic pressure, and ocular perfusion pressure [11]. Remarkably, the magnitudes of both the ERG and PERG amplitudes were dependent on the amount of cigarettes smoked in our study. The effects of cigarette smoking on PERG amplitudes and implicit times in habitual smokers have been previously reported [34]. There are several mechanisms by which cigarette smoking negatively affects the retina: increased oxidative stress, lipid peroxidation, and platelet aggregation, as well as a reduction in plasma high-density lipoprotein or antioxidant levels $[35,36]$. It is worth noting that WHR was negatively correlated with the rod b-wave implicit times of full-field ERG in our study. This finding indicates that obese patients had longer peak times of the rod-mediated response compared with their lean counterparts. Indeed, the retinal function of patients with hyperlipidemia was significantly decreased compared with healthy controls, even before the occurrence of pathological changes in the 
fundus, and BMI values were negatively correlated with the OP wave index [37].

Carotid arteries are the main blood vessels that deliver blood to the brain and the remaining head organs; blockage in the carotid artery decreases blood flow to the brain and the orbits. It has previously been reported that in cases of severe unilateral ICAS, the orbital and ocular blood flow velocities, visual functional, and morphological parameters have symmetrical patterns in both eyes and are generally reduced $[38,39]$. This finding has been attributed to flow redistribution via the circle of Willis, which supplies several collateral pathways that determine the balance of flow between the two cerebral hemispheres and between the anterior and posterior cerebral circulations [40]. The presence of collateral circulatory pathways is crucial to maintain the cerebral perfusion pressure, metabolism, and function. The external carotid artery via reversed flow in the ophthalmic artery and leptomeningeal anastomoses further contribute to the collateral circulation [40]. This finding is in concordance with our observations in which no differences were identified in the PERG, PVER, or full-field ERG wave amplitudes or implicit times between the EIS and fellow eyes of patients with unilateral significant ICAS. Similarly, there were no discrepancies in the retinal morphology in the OCT images and IOP between both eyes of the same patient with severe ICAS. These findings may indicate that a reduction in the blood inflow to the head as a result of unilateral ICAS affects the hemodynamic blood flow conditions both in the ipsilateral and fellow eyes.

Reliable measurement of retinal function is needed to provide information beyond that which could be obtained by routine ophthalmological examination. It has been proved that ERG responses ensure a good function of whole retina, and the mfERG assesses the macular function. Recently, visual electrophysiological tests were proposed to evaluate preoperative macular function, which is used to predict the outcome of cataract surgery [41] or as a viable indicator of drug penetration into the central nervous system through the blood-neural barriers [42]. Our findings would support the notion of using the ERG testing also as a new biomarker to assess retinal blood flow conditions that can be affected by hemodynamically significant ICAS in apparently healthy eyes. Thus, preoperative visual electrophysiological tests would be essential for determining whether and when the carotid surgery is necessary for asymptomatic ICAS.

A methodological limitation of our study design was the analysis of both eyes for each control to achieve maximal statistical power for comparison with EIS and ECS eyes. Due to the fact that parameters of electrophysiological function for the right and left eyes of the same control were not independent, such procedure might overestimate statistical significance of differences between ICAS and non-ICAS eyes. Unfortunately, we could not use relevant parametric tests to adjust for the correlations between both eyes of each subject since, as noted above, distributions of the parameters deviated from normal distribution. The procedure where each eye is treated as a separate item for the statistical analysis is widely used in ophthalmology in spite of the methodological objections.

\section{Conclusions}

Our study demonstrated that retinal bioelectrical function is negatively affected by hemodynamically significant ICAS despite the absence of the objective clinical signs and symptoms of ocular ischemia. ERG and PERG testing appear to be valuable tools for the detection of retinal ischemia as a result of severe carotid stenosis and may represent a clinically useful adjunct to standard algorithms in the management of asymptomatic ICAS patients, particularly in the consideration of carotid surgery.

\section{Conflicts of Interest}

The authors declare that there is no conflict of interest regarding the publication of this paper.

\section{Authors' Contributions}

Anna Machalińska and Aleksandra Kowalska-Budek contributed equally to this study.

\section{Acknowledgments}

This work was supported by the Polish National Science Center Grant no. UMO-2012/05/B/NZ7/02299 (to Miłosz Piotr Kawa).

\section{References}

[1] M. D. Walker, J. R. Marler, M. Goldstein et al., "Endarterectomy for asymptomatic carotid artery stenosis. Executive Committee for the Asymptomatic Carotid Atherosclerosis Study," Jama, vol. 273, no. 18, pp. 1421-1428, 1995.

[2] H. J. Barnett, M. Eliasziw, H. E. Meldrum, and D. W. Taylor, "Do the facts and figures warrant a 10-fold increase in the performance of carotid endarterectomy on asymptomatic patients?" Neurology, vol. 46, no. 3, pp. 603-608, 1996.

[3] F. Block and M. Schwarz, "The b-wave of the electroretinogram as an index of retinal ischemia," General Pharmacology, vol. 30, no. 3, pp. 281-287, 1998.

[4] L. Wachtmeister, "Oscillatory potentials in the retina: what do they reveal," Progress in Retinal and Eye Research, vol. 17, no. 4, pp. 485-521, 1998.

[5] R. Siliprandi, M. G. Bucci, R. Canella, and G. Carmignoto, "Flash and pattern electroretinograms during and after acute intraocular pressure elevation in cats," Investigative Ophthalmology \& Visual Science, vol. 29, no. 4, pp. 558-565, 1988.

[6] J. D. Dugan and W. R. Green, "Ophthalmologic manifestations of carotid occlusive disease," Eye (London, England), vol. 5, no. Pt 2, pp. 226-238, 1991.

[7] E. Mendrinos, T. G. Machinis, and C. J. Pournaras, "Ocular ischemic syndrome," Survey of Ophthalmology, vol. 55, no. 1, pp. 2-34, 2010.

[8] B. L. Thiele, J. V. Young, P. M. Chikos, J. H. Hirsch, and D. E. Strandness, "Correlation of arteriographic findings and symptoms in cerebrovascular disease," Neurology, vol. 30, no. 10, pp. 1041-1046, 1980.

[9] G. C. Brown and L. E. Magargal, "The ocular ischemic syndrome. Clinical, fluorescein angiographic and carotid 
angiographic features," International Ophthalmology, vol. 11, no. 4, pp. 239-251, 1988.

[10] K. Coleman, D. Fitzgerald, P. Eustace, and D. Bouchier-Hayes, "Electroretinography, retinal ischaemia and carotid artery disease," European Journal of Vascular Surgery, vol. 4, no. 6, pp. 569-573, 1990.

[11] P. K. Kofoed, I. C. Munch, S. K. Holfort et al., "Cone pathway function in relation to asymmetric carotid artery stenosis: correlation to blood pressure," Acta Ophthalmologica, vol. 91, no. 8, pp. 728-732, 2013.

[12] D. C. Hood, "Assessing retinal function with the multifocal technique," Progress in Retinal and Eye Research, vol. 19, no. 5, pp. 607-646, 2000.

[13] G. E. Holder, "Pattern electroretinography (PERG) and an integrated approach to visual pathway diagnosis," Progress in Retinal and Eye Research, vol. 20, no. 4, pp. 531-561, 2001.

[14] J. J. Ricotta, A. Aburahma, E. Ascher et al., "Updated Society for Vascular Surgery guidelines for management of extracranial carotid disease: executive summary," Journal of Vascular Surgery, vol. 54, no. 3, pp. 832-836, 2011.

[15] E. G. Grant, C. B. Benson, G. L. Moneta et al., "Carotid artery stenosis: gray-scale and Doppler US diagnosis-Society of Radiologists in ultrasound consensus conference," Radiology, vol. 229, no. 2, pp. 340-346, 2003.

[16] C. P. Oates, A. R. Naylor, T. Hartshorne et al., "Joint recommendations for reporting carotid ultrasound investigations in the United Kingdom," European Journal of Vascular and Endovascular Surgery, vol. 37, no. 3, pp. 251-261, 2009.

[17] D. L. McCulloch, M. F. Marmor, M. G. Brigell et al., "ISCEV standard for full-field clinical electroretinography (2015 update)," Documenta Ophthalmologica, vol. 130, no. 1, pp. 1-12, 2015.

[18] M. Bach, M. G. Brigell, M. Hawlina et al., "ISCEV standard for clinical pattern electroretinography (PERG): 2012 update," Documenta Ophthalmologica, vol. 126, no. 1, pp. 1-7, 2013.

[19] J. V. Odom, M. Bach, M. Brigell et al., "ISCEV standard for clinical visual evoked potentials: (2016 update)," Documenta Ophthalmologica, vol. 133, no. 1, pp. 1-9, 2016.

[20] P. Törnquist and A. Alm, "Retinal and choroidal contribution to retinal metabolism in vivo. A study in pigs," Acta Physiologica Scandinavica, vol. 106, no. 3, pp. 351-357, 1979.

[21] M. Post, W. Goslawski, M. Modrzejewska, M. Wielusinski, J. Kazmierczak, and W. Lubinski, "Electrophysiological function of the retina and optic nerve in patients with atrial fibrillation," Documenta Ophthalmologica, vol. 131, no. 1, pp. 53-62, 2015.

[22] N. Sayin, N. Kara, F. Uzun, and I. F. Akturk, "A quantitative evaluation of the posterior segment of the eye using spectral-domain optical coherence tomography in carotid artery stenosis: a pilot study," Ophthalmic Surgery, Lasers and Imaging Retina, vol. 46, no. 2, pp. 180-185, 2015.

[23] N. Utsugi, K. Takahashi, and S. Kishi, "Choroidal vascular occlusion in internal carotid artery obstruction," Retina, vol. 24, no. 6, pp. 915-919, 2004.

[24] J. Ahmed, M. K. Pulfer, and R. A. Linsenmeier, "Measurement of blood flow through the retinal circulation of the cat during normoxia and hypoxemia using fluorescent microspheres," Microvascular Research, vol. 62, no. 2, pp. 143-153, 2001.

[25] A. Bill and G. O. Sperber, "Control of retinal and choroidal blood flow," Eye (London, England), vol. 4, no. Pt 2, pp. 319-325, 1990.
[26] P. K. Kofoed, I. C. Munch, B. Sander et al., "Prolonged multifocal electroretinographic implicit times in the ocular ischemic syndrome," Investigative Ophthalmology \& Visual Science, vol. 51, no. 4, pp. 1806-1810, 2010.

[27] C. J. Karwoski and X. Xu, "Current source-density analysis of light-evoked field potentials in rabbit retina," Visual Neuroscience, vol. 16, no. 2, pp. 369-377, 1999.

[28] L. M. Haugh, R. A. Linsenmeier, and T. K. Goldstick, "Mathematical models of the spatial distribution of retinal oxygen tension and consumption, including changes upon illumination," Annals of Biomedical Engineering, vol. 18, no. 1, pp. 19-36, 1990.

[29] C. E. Riva, J. E. Grunwald, and B. L. Petrig, "Reactivity of the human retinal circulation to darkness: a laser Doppler velocimetry study," Investigative Ophthalmology \& Visual Science, vol. 24, no. 6, pp. 737-740, 1983.

[30] G. T. Feke, R. Zuckerman, G. J. Green, and J. J. Weiter, "Response of human retinal blood flow to light and dark," Investigative Ophthalmology \& Visual Science, vol. 24, no. 1, pp. 136-141, 1983.

[31] L. Maffei and A. Fiorentini, "Generator sources of the pattern ERG in man and animals," in Frontiers in clinical neuroscience, R. Q. Cracco and I. Bodis-Wollner, Eds., vol. 3, pp. 101-116, Wiley-Liss, New York, 1986.

[32] E. Lenassi, A. G. Robson, M. Hawlina, and G. E. Holder, "The value of two-field pattern electroretinogram in routine clinical electrophysiologic practice," Retina, vol. 32, no. 3, pp. 588-599, 2012.

[33] Y. J. Sepah, M. A. Sadiq, M. Hassan et al., "Assessment of retinal structural and functional characteristics in eyes with autoimmune retinopathy," Current Molecular Medicine, vol. 15, no. 6, pp. 578-586, 2015.

[34] F. C. Gundogan, A. H. Durukan, T. Mumcuoglu, G. Sobaci, and M. Z. Bayraktar, "Acute effects of cigarette smoking on pattern electroretinogram," Documenta Ophthalmologica, vol. 113, no. 2, pp. 115-121, 2006.

[35] Group A-REDSR, "Risk factors associated with age-related macular degeneration. A case-control study in the agerelated eye disease study: age-related eye disease study report number 3," Ophthalmology, vol. 107, no. 12, pp. 2224-2232, 2000.

[36] S. S. Ni Dhubhghaill, M. T. Cahill, M. Campbell, L. Cassidy, M. M. Humphries, and P. Humphries, "The pathophysiology of cigarette smoking and age-related macular degeneration," Advances in Experimental Medicine and Biology, vol. 664, pp. 437-446, 2010.

[37] B. Zhang, Q. Qiu, L. Yin, Y. Yao, C. Wang, and X. Wu, "Measurement of retinal function with flash-electroretinography in Chinese patients with hyperlipidemia," Graefe's Archive for Clinical and Experimental Ophthalmology, vol. 252, no. 9, pp. 1385-1392, 2014.

[38] H. Heßler, H. Zimmermann, T. Oberwahrenbrock et al., "No evidence for retinal damage evolving from reduced retinal blood flow in carotid artery disease," BioMed Research International, vol. 2015, Article ID 604028, p. 8, 2015.

[39] V. P. Kozobolis, E. T. Detorakis, G. S. Georgiadis, A. A. Achtaropoulos, T. T. Papas, and M. K. Lazarides, "Perimetric and retrobulbar blood flow changes following carotid endarterectomy," Graefe's Archive for Clinical and Experimental Ophthalmology, vol. 245, no. 11, pp. 1639$1645,2007$. 
[40] E. M. Vriens, G. H. Wieneke, B. Hillen, B. C. Eikelboom, A. C. Van Huffelen, and G. H. Visser, "Flow redistribution in the major cerebral arteries after carotid endarterectomy: a study with transcranial Doppler scan," Journal of Vascular Surgery, vol. 33, no. 1, pp. 139-147, 2001.

[41] J. An, L. Zhang, Y. Wang, and Z. Zhang, "The success of cataract surgery and the preoperative measurement of retinal function by electrophysiological techniques," Journal of Ophthalmology, vol. 2015, Article ID 401281, p. 10, 2015.

[42] J. Charng, Z. He, A. J. Vingrys et al., "Retinal electrophysiology is a viable preclinical biomarker for drug penetrance into the central nervous system," Journal of Ophthalmology, vol. 2016, Article ID 5801826, p. 12, 2016. 


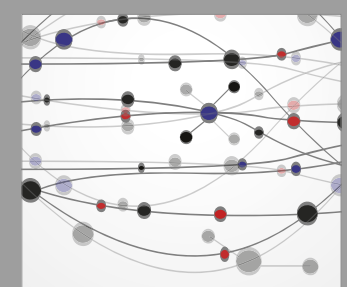

The Scientific World Journal
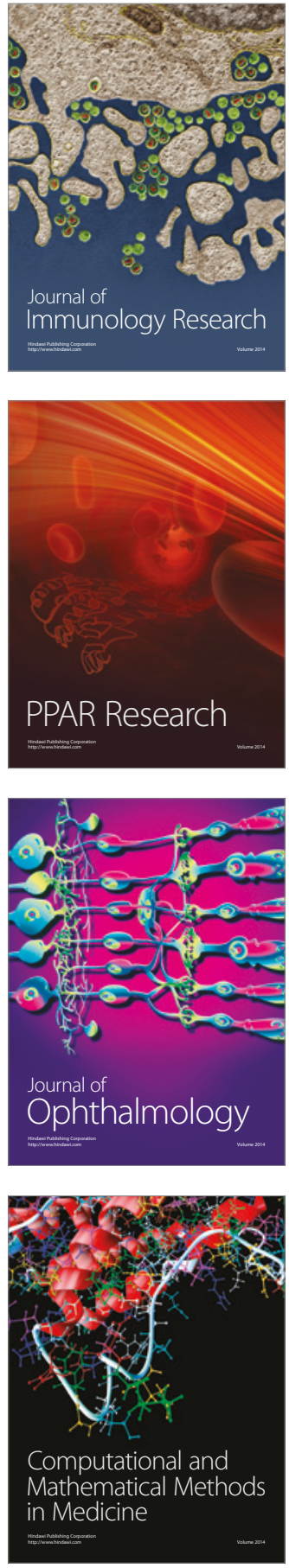

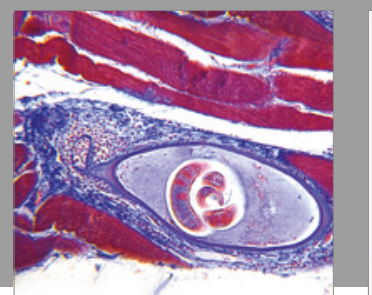

Gastroenterology Research and Practice
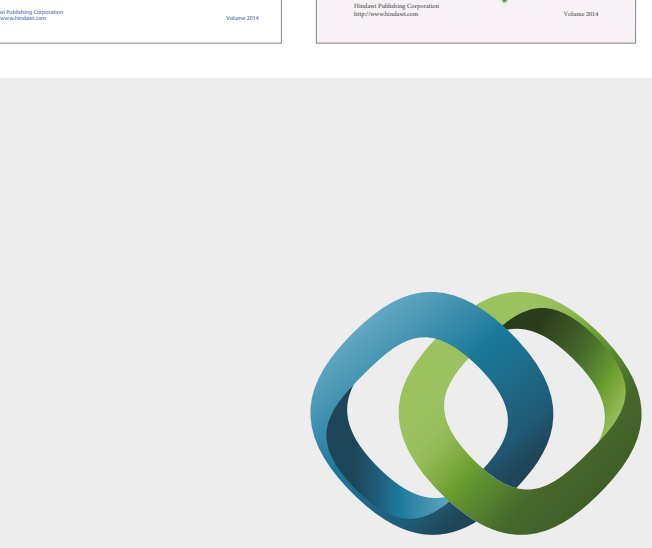

\section{Hindawi}

Submit your manuscripts at

https://www.hindawi.com
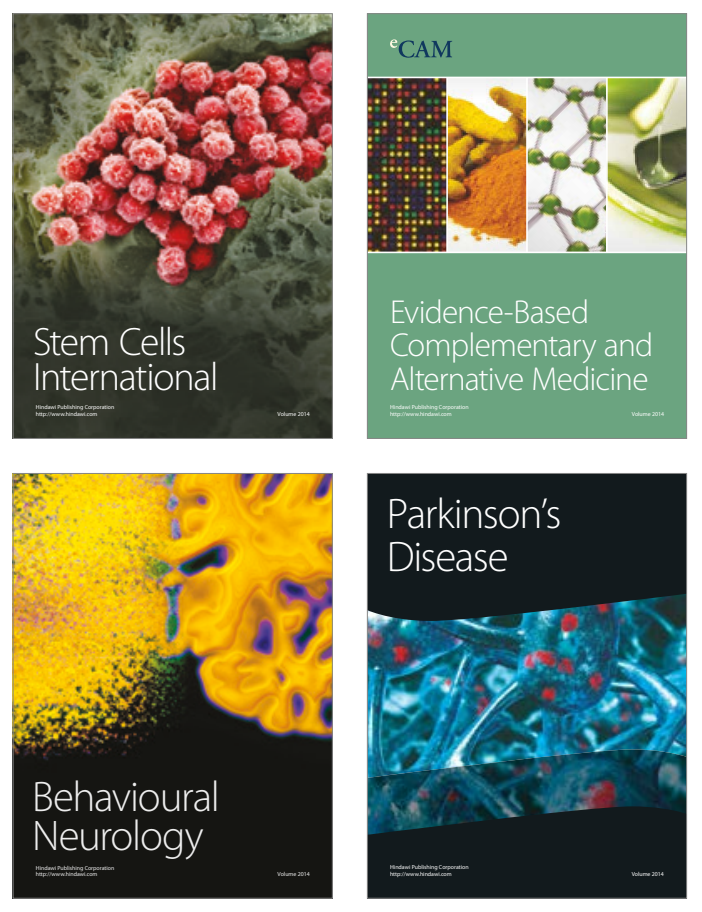
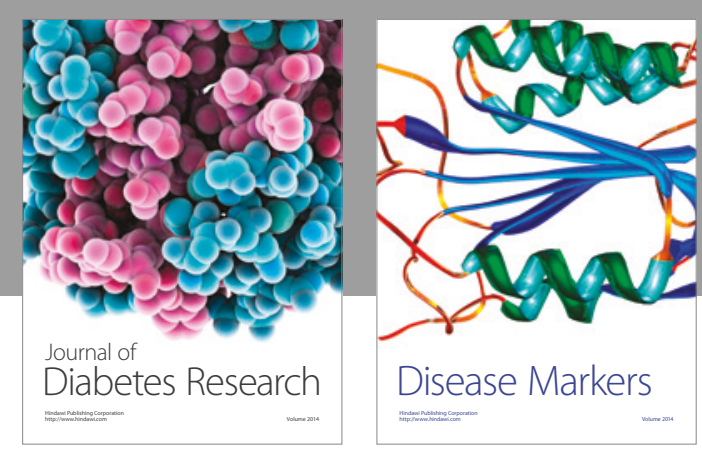

Disease Markers
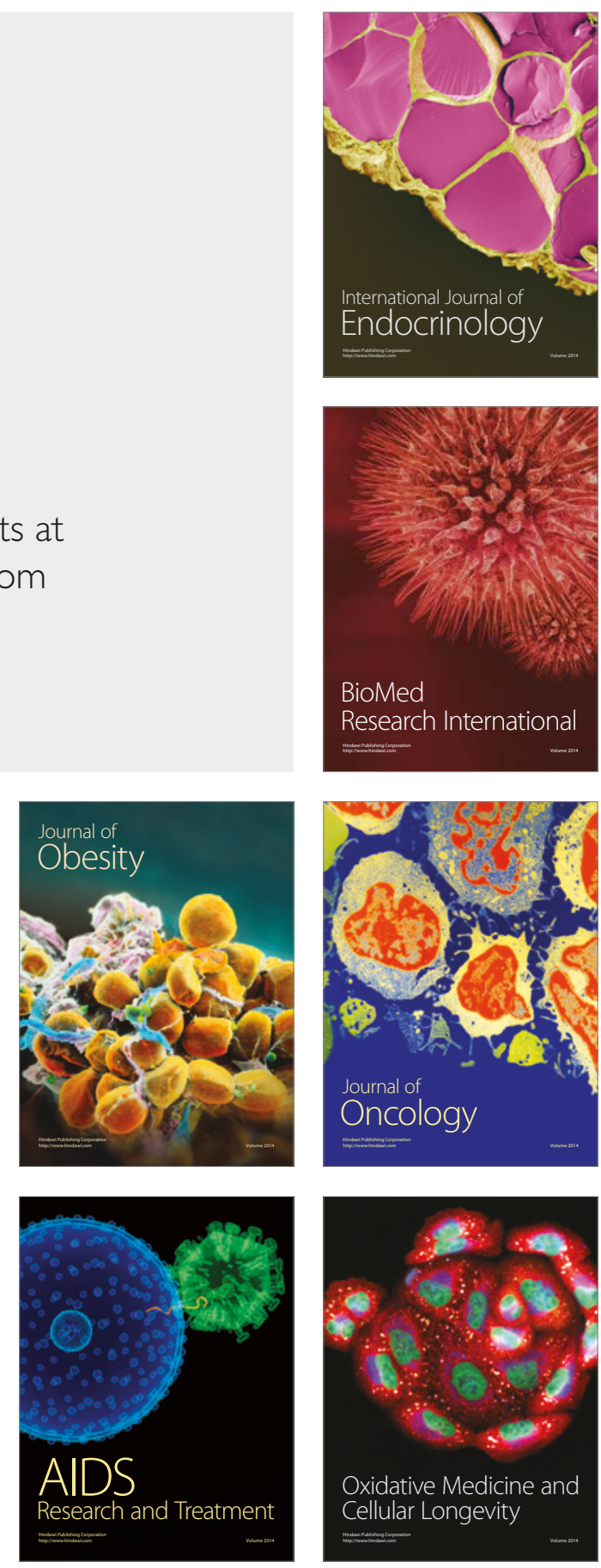